\title{
Archéopages
}

Archéopages

Archéologie et société

$44 \mid 2017$

Terrains vagues

\section{L'outillage lithique en contexte de transformation des métaux}

\section{Méthodologie et perspectives}

Lithic tooling in the context of metal processing. Methodology and perspectives

Las herramientas líticas en un contexto de transformación de los metales.

Metodología y perspectivas

\section{Maxence Pieters}

\section{(2) OpenEdition}

\section{Journals}

Édition électronique

URL : https://journals.openedition.org/archeopages/1567

DOI : 10.4000/archeopages. 1567

ISSN : 2269-9872

\section{Éditeur}

INRAP - Institut national de recherches archéologiques préventives

\section{Édition imprimée}

Date de publication : 1 juin 2017

Pagination : 76-85

ISSN : 1622-8545

\section{Référence électronique}

Maxence Pieters, "L'outillage lithique en contexte de transformation des métaux », Archéopages [En ligne], 44 | 2017, mis en ligne le 01 juillet 2019, consulté le 04 juin 2021. URL : http:// journals.openedition.org/archeopages/1567; DOI : https://doi.org/10.4000/archeopages.1567 


\section{L'outillage lithique en contexte de transformation des métaux Méthodologie et perspectives}

Maxence Pieters Centre ardennais de recherche archéologique, UMR 6298, «ArTeHiS »

\section{6}

L'outillage lithique découvert lors des fouilles de sites métallurgiques reste, malgré des corpus parfois considérables, insuffisamment pris en compte. L'analyse fonctionnelle fine de ces outils permet pourtant d'obtenir des informations souvent inaccessibles par l'étude des fabricats métalliques. Chaque catégorie fonctionnelle (supports de frappe, abrasifs, brunissoirs, outils

En 1996, dans son ouvrage de synthèse sur l'artisanat, Jean-Paul Guillaumet mettait pour la première fois en avant la problématique de l'outillage lithique : « Il reste à prendre en compte dans ces ateliers de transformation des métaux les témoins oubliés lors de la fouille et ce qui est irrémédiablement perdu. Dans la première catégorie, on peut citer les pièces lithiques utilisées comme lissoir, broyon, tas, pierre à aiguiser, poids, contrepoids et cales et souvent considérées comme des cailloux du terrain naturel. Dans la seconde, il faut mettre les outils, instruments et établis en matériaux périssables, bois, cuir, boyaux et la plupart de ceux en matériaux réutilisables, métaux précieux, bronze, fer et plomb » (Guillaumet, 1996). Cette mise en parallèle des outils lithiques avec les éléments périssables disparus illustre bien l'absence de prise en compte de l'outillage lithique. Rares sont les sites dans lesquels plus de quelques outils en pierre sont découverts et les corpus ne sont jamais analysés. Tout au plus font-ils l'objet de planches, parfois commentées, mettant en d'aiguisage et outils de broyage) nécessite une approche spécifique qui ouvre de nombreuses perspectives pour une meilleure compréhension aussi bien des processus métallurgiques que des modalités d'exploitation du milieu, des circuits d'échanges ou encore de la connaissance par les fabricants des propriétés des roches.

évidence l'absence de méthodologie pour une analyse problématisée de ce mobilier (par exemple Jacobi, 1974).

Dans les années 1990, la fouille du Lycée militaire à Autun, bientôt suivie de la publication du site (Chardron-Picault, Pernault, 1999), met en évidence l'importance de l'outillage lithique lors des opérations de transformation des métaux. Ce sont au total 252 outils qui sont découverts lors de la fouille, du plus petit ( $0,3 \mathrm{~g}$ pour un abraseur en pierre ponce) au plus gros (892 $\mathrm{kg}$ pour une enclume lourde en grès). Cet outillage fait l'objet d'une ébauche d'analyse, avec une première tentative de classification typologique du petit outillage. Cette découverte exceptionnelle, du fait d'une collecte systématique et efficace du mobilier lithique, n'a malheureusement pas engendré d'intérêt scientifique dans les années qui ont suivi. Pourtant, l'expérience a montré depuis que de tels ensembles mobiliers sont courants en contexte de métallurgie de transformation. L'absence d'outils en pierre constitue au contraire une anomalie, qui 
peut être imputée aux conditions de conservation du site, aussi bien qu'à l'absence de formation des archéologues vis-à-vis de ce mobilier.

C'est à cette situation particulière - une documentation archéologique abondante mais non publiée et une absence totale de méthodologie adaptée - que nous nous sommes confronté à partir de 2009, d'abord dans le cadre d'un travail universitaire sous la direction de Jean-Paul Guillaumet (Pieters, 2013), puis à travers différentes études pour des fouilles programmées ou préventives. Cette friche scientifique a fourni l'opportunité de développer une méthode d'approche originale, adaptée aux spécificités de l'outillage lithique, tout en gardant pour principe de conserver une démarche qui ne soit pas strictement conçue pour la pierre. Car la spécificité de l'outillage lithique utilisé pour la transformation des métaux est que la plupart des catégories fonctionnelles identifiées existent également dans d'autres matériaux que la pierre, notamment le métal et la céramique.

La méthodologie mise au point et expérimentée sur de nombreux corpus d'étude découle à la fois d'une approche empirique du mobilier (lecture des informations perceptibles sur l'objet, croisement des données accumulées, comparaison avec l'outillage moderne ou contemporain...) et d'une démarche conceptuelle, rendue nécessaire par les questionnements spécifiques engendrés par un mobilier qui se différencie parfois peu des éléments naturels.

\section{La reconnaissance de l'outillage lithique}

La difficulté de la reconnaissance des outils lithiques lors de la fouille tient aux particularités de leur conception. Le matériau utilisé est par essence naturel. Seuls trois indices permettent de distinguer un outil d'un écofact : la mise en forme, les traces d'utilisation et la nature allochtone de la roche, trahissant un déplacement anthropique du matériau. Toutefois, l'expérience démontre qu'aucun de ces indices n'est déterminant [cf. encadré].

\section{Indices de mise en forme}

La mise en forme de la pierre est de toute évidence un indice flagrant pour distinguer un outil d'une pierre naturelle. Toutefois, la majeure partie de l'outillage lithique n'est pas mise en forme, ou alors de façon très partielle. Il s'agit alors simplement d'une préparation des surfaces actives, sans recherche d'une forme particulière. Chaque outil est ainsi pratiquement unique.

De plus, nombre d'outils mis en forme ont un aspect peu différent des pierres naturelles. C'est le cas de nombreux broyons, dont la forme se rapproche de celle des galets. La forme arrondie de l'outil et ses surfaces lisses, voire polies, engendrent une certaine confusion. Accessoirement, les traces d'utilisation sur une pierre peuvent aisément être confondues avec une mise en forme. Ainsi, de nombreux broyons découverts en contexte métallurgique ont été interprétés comme des tas (Chardron-Picault, Pernault, 1999).

La mise en forme n'est donc pas une condition nécessaire pour reconnaître un outil. Elle définit pourtant traditionnellement la notion même d'objet, mais n'est pas pertinente pour l'outillage lithique des âges des métaux, de la même manière qu'on ne peut nier le caractère d'outils aux percuteurs préhistoriques, quand bien même ils ne sont pas mis en forme. D'autres arguments pour identifier un outil sont donc nécessaires.

\section{Les indices d'utilisation}

Les traces d'utilisation peuvent être considérées comme un recours possible pour identifier des outils non mis en forme. Elles varient suivant le type d'outil, généralement des traces de friction ou de percussion et, dans quelques cas de figure, de friction et de percussion. L'écueil le plus fréquent est la confusion possible avec des traces de mise en forme ou une altération naturelle ou accidentelle de la pierre. L'érosion naturelle altère les surfaces exposées aux intempéries ou au passage de l'eau (cours d'eau charriant des particules abrasives ou bord de mer provoquant friction et percussion des éléments lithiques entre eux). De même, l'interaction entre l'homme et l'environnement peut engendrer des altérations sur des éléments lithiques, sans pour autant qu'ils puissent être considérés comme des outils. Enfin, une trace d'utilisation isolée, témoignant d'une unique utilisation, n'est pas suffisante pour identifier un outil.

Au delà de ces difficultés d'identification, nombre d'outils ne portent aucune trace d'utilisation. Deux cas de figure peuvent se rencontrer. Le premier est celui des outils que l'on peut considérer comme des consommables, tels les abrasifs. Ces outils ont une durée d'utilisation limitée, du fait d'une usure rapide. Si les outils les plus gros, comme les tables abrasives, peuvent subir une réfection de surface qui prolonge leur durée de vie, d'autres sont jetés dès que l'ensemble de leur surface a été utilisée. C'est le cas de abraseurs, dont la taille réduite ne permet pas d'envisager une réfection. Ils sont donc stockés en quantité. Ainsi, le site du Lycée militaire à Autun a livré un ensemble de 60 abrasifs réunis dans une fosse. Une partie de ces outils étaient utilisés, d'autres prêts à l'emploi et les derniers n'étaient que des fragments de grès en cours de mise en forme. Le second cas de figure est celui de certains outils de bonne qualité, dont la surface ne porte pas de traces malgré une utilisation prononcée. C'est notamment le cas des supports de frappe et plus particulièrement des matrices de chaudronnerie. Les contraintes mécaniques subies par ces outils sont insuffisantes pour en marquer la surface. Le métal chaud ou recuit forme un tampon entre la pierre et le marteau ou la masse, amortissant les chocs. 


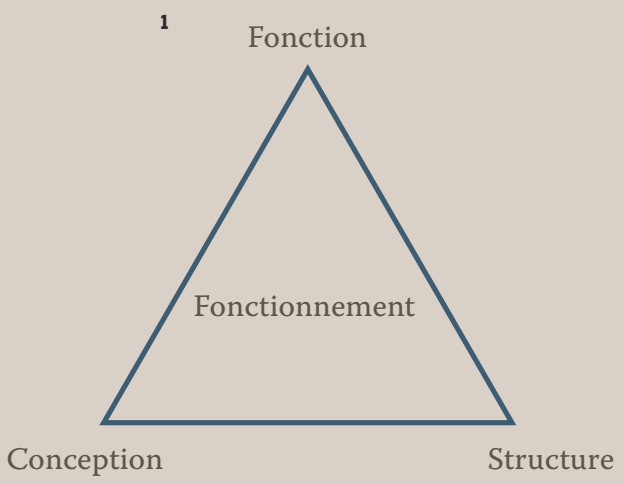

Le principe de l'identification fonctionnelle L'outillage lithique illustre bien les limites de la définition traditionnelle de l'outil en particulier et de l'objet en général. La notion de fabrication, essentielle dans la définition classique de l'objet, ne peut s'appliquer dans ce cas de figure, puisque nombre d'outils ne sont pas mis en forme. De plus, le stockage d'ébauches ou de matière première destinées à servir d'abrasifs montre qu'avant même la mise en forme ou l'utilisation, ces pierres sont considérées comme des outils. Une question se pose donc : à partir de quel moment peut-on parler d'outil, puisqu'aucun indice ne peut être considéré comme déterminant ? La réponse à cette question nécessite une conceptualisation de la notion d'objet, puisqu'une définition physique est impossible. L'outil n'est pas le propre de l'homme, puisqu'on le retrouve dans le monde animal, sous la forme d'éléments naturels utilisés bruts - pierres pour ouvrir les bogues chez les chimpanzés ou pour ouvrir les coquillages chez les loutres - ou transformés - feuilles découpées pour chasser les insectes chez les corneilles (Bluff et al., 2007). Une différence notable semble toutefois exister entre le comportement humain et celui des autres animaux : la conservation de l'outil pour des utilisations futures. Par là même, l'Homme attribue une fonction à l'outil, qui ne se perd qu'une fois l'objet devenu inutilisable. Ainsi, c'est la fonction qui fait l'objet et non sa fabrication, ni même son utilisation. Flle peut être définie assez simplement comme le rôle attribué à l'objet, technique (fonction physique) et/ou symbolique. La fonction symbolique traduit physiquement une idée. Ainsi, une pièce symbolise une valeur d'échange, le vêtement symbolise l'appartenance à un groupe social/ethnique en plus de sa fonction technique d'abriter le corps du froid, de la pluie ou des rayons du soleil.

De cette fonction découle la conception de l'objet : celui-ci est élaboré mentalement pour pouvoir répondre à la fonction à laquelle il est destiné, avant d'être fabriqué ou d'utiliser directement un élément naturel adapté. Cette notion de conception est totalement différente de celle d'invention ou d'innovation (sur ces notions, voir Beaune, 2008). La structure de l'objet en découle, comprise comme l'ensemble des parties qui le constituent et de leurs interactions : zones de préhension ou de fixation, corps, surfaces actives... Fonction, conception et structure de l'objet sont ainsi indissolublement liées. Flles délimitent le cadre de fonctionnement de l'objet qui, s'il peut être utilisé différemment de ce pourquoi il est conçu, ne peut déborder du champ des possibles défini par sa structure [ill. 1]. Ainsi, si un marteau à frapper devant-lourd

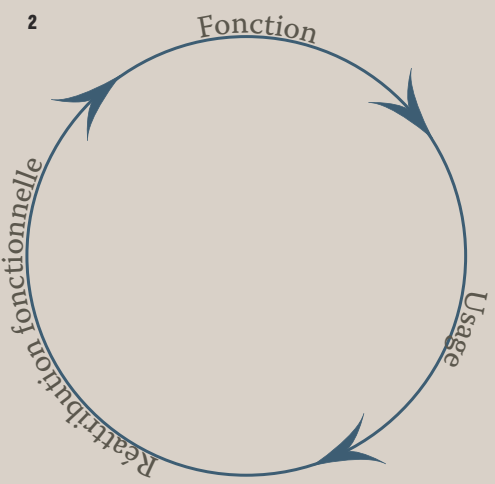

1.Schématisation du concept de fonction. 2.Schématisation du cycle de vie de l'objet. marteau de forge pesant 3 à $5 \mathrm{~kg}$ - peut être utilisé pour enfoncer un pieu, une enclume ne peut faire le même usage, du fait de sa masse trop importante. Ici apparaît la notion d'usage qui s'applique à la phase d'utilisation de l'objet. Cette notion est d'autant plus complexe qu'elle prend en compte non seulement l'utilisation proprement dite de l'objet, mais également le contexte dans lequel elle a lieu. Ainsi, l'usage d'un marteau de menuisier n'est pas le même dans un atelier de menuiserie que dans une habitation : usage professionnel d'un côté et bricolage de l'autre. L'usage d'un objet est théoriquement défini par sa fonction, mais celle-ci ne le limite pas. Trois niveaux d'utilisation peuvent être définis : l'usage approprié, où l'outil est utilisé conformément à sa fonction (marteau à frapper devant utilisé pour forger); l'usage détourné, lorsque l'outil est utilisé sans tenir compte de sa fonction, mais en respectant son fonctionnement (marteau à frapper devant utilisé pour enfoncer un piquet de clôture) ; l'usage contrarié, quand l'outil est utilisé sans tenir compte de sa fonction et sans respecter son fonctionnement normal (tenailles utilisées pour mater un clou revêche à l'arrachage). L'usage de l'objet peut varier au cours du temps, sans pour autant remettre en cause sa fonction. Toutefois, on observe dans certains cas de figure une remise en cause définitive de la fonction de 1'outil. On ne peut alors plus parler d'usage, mais de réattribution fonctionnelle. Cette dernière notion intervient lorsque l'objet perd de façon définitive sa fonction, qu'il devienne inutilisable (bris) ou inutile. La réattribution fonctionnelle la plus courante est celle qui voit les objets devenir déchets, puisque la plupart sont découverts en contexte détritique. Se pose alors la question fondamentale des causes de ce phénomène. La gestion des déchets est différente suivant les cultures, avec une pratique plus ou moins avancée du remploi, de la réutilisation et du recyclage, qui relèvent fondamentalement de pratiques économiques : l'absence de recyclage des métaux témoigne ainsi de leur abondance.

Fonction, usage et réattribution fonctionnelle permettent ainsi de conceptualiser le cycle de l'existence de l'objet, qui ne s'interrompt qu'avec sa disparition [ill. 2]. D'un point de vue pratique, ce cadre de raisonnement invite à considérer systématiquement l'objet dans toute sa complexité et non pas seulement dans le dernier état de son utilisation, afin de mieux comprendre l'évolution des activités sur un site donné, l'importance culturelle de l'objet (qui peut devenir dépôt ou offrande), mais également le système économique général dans lequel il peut s'intégrer. 
L'expérimentation a montré que, sur un matériau de qualité, seuls les seuls coups directs portés à la surface de l'outil peuvent altérer cette dernière (Pieters, 2010).

Enfin, les traces d'utilisation, si elles peuvent être examinées pour identifier un outil, doivent être considérées avec la plus grande prudence pour leur identification fonctionnelle. Hormis la confusion entre traces de mise en forme et traces d'utilisation, il faut tenir compte d'un phénomène récurrent : la présence de traces d'utilisation ne correspondant pas à la fonction de l'outil. Elles témoignent d'une utilisation à contre-emploi ou de réutilisations possibles de l'outil et peuvent engendrer une certaine confusion.

\section{Les matériaux allochtones}

Le dernier indice disponible pour l'identification d'un outil lithique est la nature de la pierre elle-même. Dans un contexte géologique donné, les indices de déplacement des pierres renseignent sur leur utilisation. Toute pierre allochtone, non présente naturellement sur un site, est potentiellement un objet. Deux arguments permettent de distinguer les roches allochtones : leur nature lithologique et leur morphologie. Les exemples sont nombreux : présence de roches sédimentaires sur des massifs primaires, de galets en sommet de relief... Cette attention à la présence de roches allochtones est d'autant plus importante qu'elles forment fréquemment l'essentiel du matériau employé pour la réalisation des outils. L'exemple de l'oppidum de Bibracte (Saône-et-Loire) est révélateur, avec une proportion de $75 \%$ d'outils réalisés dans des pierres allochtones.

L'investissement dans la matière première peut être relativement important, puisqu'on observe fréquemment des approvisionnements distants de plusieurs dizaines, voire plusieurs centaines de kilomètres. Le matériau le plus révélateur de ces pratiques est la pierre ponce. Elle est très fréquente sur les sites de transformation du métal et ce dès l'âge du Bronze, comme le montre l'exemple du site de Fort-Harrouard à Sorel-Moussel (Eure-et-Loir), où de nombreux exemplaires d'abrasifs réalisés dans ce matériau ont été découverts (Mohen, Bailloud, 1987). La fréquence des découvertes est telle à La Tène $\mathrm{D}$ et à l'époque romaine que l'absence de ponce sur un site de transformation des métaux peut être considérée comme une anomalie. Ce phénomène s'explique aisément par la qualité du matériau, considéré jusqu’à la fin du XVIII ${ }^{\mathrm{e}}$ siècle comme le meilleur abrasif (Landrin, 1835).

Néanmoins, la détermination de la provenance de la roche nécessite une connaissance approfondie de la géologie locale. Certains contextes sont particulièrement complexes, notamment les terrasses fossiles. Les cours d'eau peuvent déplacer les pierres sur de longues distances et les déposer sur des terrains où elles apparaissent comme des anomalies. A contrario, des éléments lithiques peuvent être déplacés sur les sites pour d'autres raisons, comme lors de l'amendement de terre à l'aide de limon (communication orale Matthieu Demierre).

\section{Identification fonctionnelle de l'outillage lithique}

Si la provenance des roches et les indices de mise en forme ou d'utilisation sont suffisants pour permettre une première discrimination sur le terrain, ils ne permettent pas une identification fonctionnelle. Celle-ci nécessite une analyse de la structure de l'outil, pour en comprendre la conception, et par là la fonction. L'analyse de la structure tient compte de cinq critères : la morphologie (identification des différentes parties fonctionnelles), les dimensions (taille et proportions de l'outil), la masse et les propriétés mécaniques des matériaux (dureté, résilience, abrasivité). L'importance respective de ces critères varie en fonction de la catégorie fonctionnelle : supports de frappe, abrasifs, brunissoirs, outils d'aiguisage ou outils de broyage.

\section{Les supports de frappe}

Les supports de frappe sont des outils fixes utilisés pour les opérations de déformation plastique des métaux. En opposant leur force d'inertie à l'énergie cinétique de l'outil travaillant en percussion, le métal est pris entre deux forces opposées, ce qui conduit à sa déformation. La forme de la surface active définit la déformation qui sera engendrée et permet de distinguer les différents types d'outils. Deux types de surfaces actives sont observées sur les supports de frappe lithiques : plane (ou plano-convexe) et concave. Ils distinguent les outils de forgeage, enclumes et tas [ill. 1], des outils d'emboutissage que sont les matrices [ill. 2]. Dans cette catégorie peuvent également prendre place les marbres, qui sont davantage des outils de réglage que de déformation plastique. Ils se distinguent par leur morphologie, avec une surface active de grande dimension, mais une faible épaisseur.

L'énergie nécessaire à la déformation du métal augmentant avec l'épaisseur de ce dernier, il existe une corrélation entre la masse du support de frappe et l'épaisseur du métal travaillé. La masse constitue ainsi le principal critère d'analyse des supports de frappe. Ces derniers peuvent être répartis en trois catégories en fonction de leur masse : légère, ordinaire et lourde. Toutefois, ces notions varient suivant la période envisagée, car les productions évoluent. Ainsi, si les enclumes lourdes de La Tène pèsent entre 50 et $200 \mathrm{~kg}$ environ, les enclumes lourdes de l'époque romaine varient entre 150 et $900 \mathrm{~kg}$.

Les supports de frappe sont des outils qui subissent des contraintes mécaniques importantes, qui peuvent se traduire de deux manières : déformation de la surface active par éclatement 

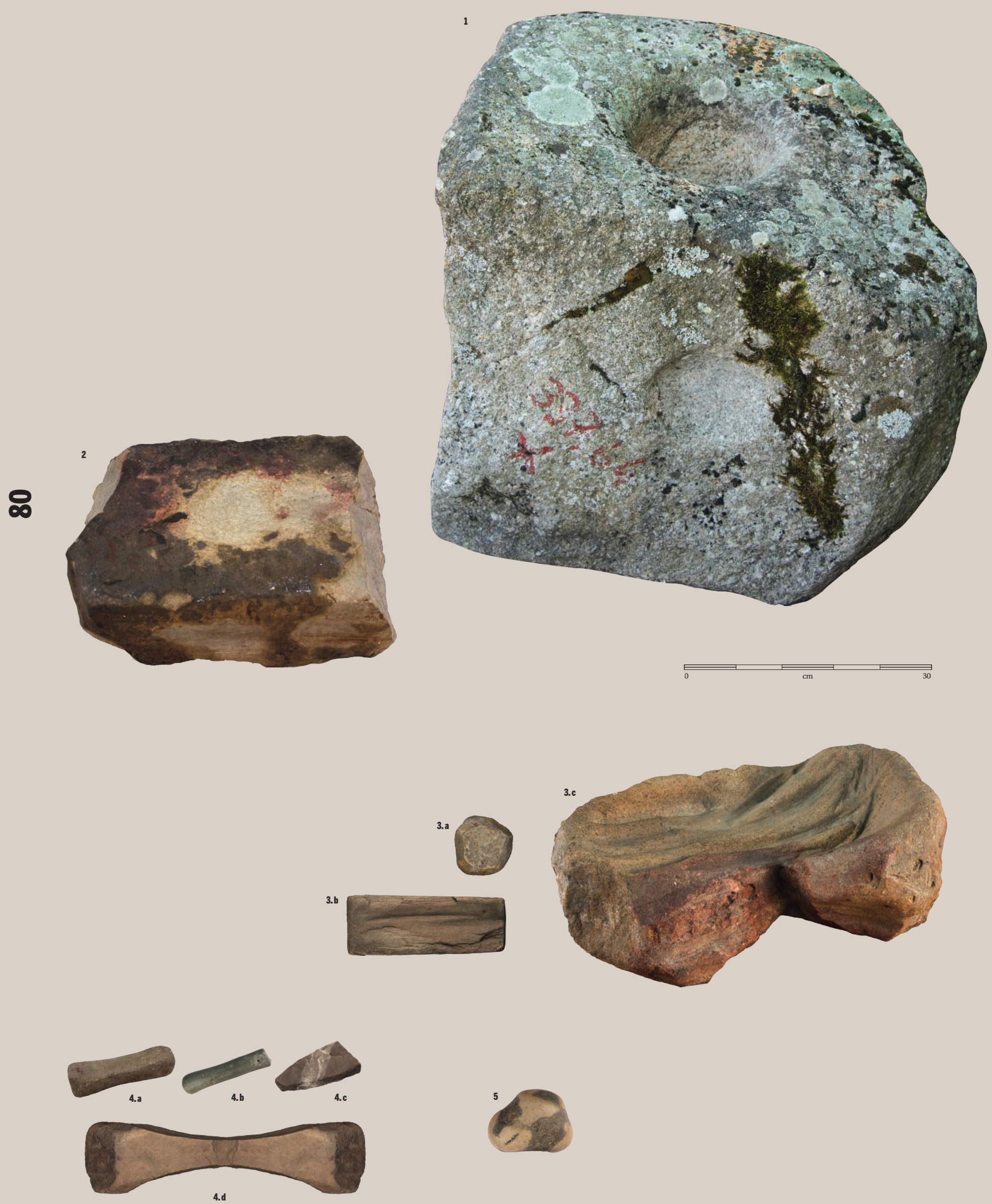
1. Enclume lourde réalisée dans un bloc brut de grès quarzitique provenant de l'atelier de bronzier de la Porte du Rebout à Bibracte. On remarque la trace laissée par les impacts et, sur la droite, la fracture réalisée lors de la réutilisation de

l'outil.

2. Matrice lourde en granite provenant du site du Lycée militaire à Autun. La dimension de l'outil permet de travailler debout. 3. Principaux types d'abrasifs : abraseur (a), tablette abrasive (b) et table abrasive (c) provenant de la fouille du Lycée militaire à Autun. Remarquer la surface creusée de la table abrasive qui résulte de réfections successives.

4. Types d'outils d'aiguisage : aiguisoir (a) et affiloir (b) provenant de Bibracte, pierre à faux (c) découverte sur le site de Stradonice et meulet (d) mis au jour lors de la fouille du Faubourg d'Arroux à Autun (dir. Stéphane Alix, Inrap). La plupart de ces outils existent dès l'âge du Bronze et sont toujours utilisés. Le meulet est un outil d'émoulage conservé à $50 \%$ et reconstitué par photomontage. 5. Brunissoir en silexite provenant de la Pâture du Couvent, Hors Couvent à Bibracte. (impacts, éclatement sous forme de cupules) ou fracture de l'outil. D'où l'importance du choix d'un matériau supportant la fatigue des chocs répétés. En l'absence d'un matériau de qualité à disposition sur les sites de production,

l'investissement consenti pour obtenir une pierre convenable peut être important, puisque l'on observe fréquemment des distances d'approvisionnement de plusieurs dizaines de kilomètres. En règle générale, il semble que ce soient les roches sédimentaires qui sont utilisées préférentiellement, essentiellement le grès, mais également certains calcaires durs, à l'exception des matrices de chaudronnerie, réalisées généralement dans des roches magmatiques (granite, rhyolite).

Le fonctionnement du support de frappe exige stabilité et longévité. Les outils de forgeage, qui subissent les plus fortes contraintes, sont systématiquement enchâssés dans le sol, ce qui leur assure une bonne stabilité et absorbe en partie les vibrations subies, augmentant leur longévité, ce qui est confirmé par l'expérimentation (Pieters, 2010). Il est donc nécessaire de porter une attention particulière à la fosse de calage de l'outil. On peut ainsi observer plusieurs fosses autour d'une enclume, témoignant du remplacement régulier de l'outil, ce qui peut permettre d'en estimer la longévité lorsque la durée d'occupation de la structure est restituable.

\section{Les abrasifs et les outils d'aiguisage}

Par définition, un abrasif est une matière minérale dure et tranchante capable d'arracher par frottement sous pression des particules d'un autre matériau. On peut ainsi considérer que les outils d'aiguisage sont des abrasifs. Par là même, les critères d'analyse qui doivent leur être appliqués sont les mêmes.

C'est le matériau qui constitue le principal critère d'analyse de ces outils. Quatre aspects sont à prendre en compte. Le premier est la nature du matériau utilisé, qui définit sa dureté. Il peut s'agir d'une roche, mais également de céramique. Le second critère est la taille des grains du matériau, dont dépend la finesse du travail sur la matière. Les méthodes habituelles de mesure du grain de la roche utilisées par les géologues sont inopérantes, car le ciment de la roche peut être lui-même abrasif. C'est donc davantage la rugosité du matériau qui doit être évaluée, à l'aide d'une échelle numérique de 1 à 7 , du grain le plus grossier au plus fin. Le grade constitue une échelle de mesure de la cohésion de la roche, notée de A (craie) à I (quartzite). Plus le grade est faible, plus les grains se renouvellent rapidement. Enfin, l'ouverture du matériau, ouvert (o) ou fermé (f), distingue les matériaux massifs des matériaux vacuolaires. Ce critère est pertinent uniquement pour la pierre ponce et le basalte, les autres matériaux étant toujours fermés.

Ces critères d'analyse du matériau, croisés avec l'expérimentation, permettent de mieux comprendre l'effet de l'abrasif sur la matière et ainsi d'associer un type d'abrasif à un matériau. Nous avons pu observer que le grès, particulièrement efficace sur le fer, est inutilisable pour le travail des cuproalliages. Son grade trop élevé empêche le renouvellement des grains. Ceux-ci s'émoussent, les creux entre les grains se comblent avec la matière et l'abrasif perd rapidement tout mordant. Au contraire, la céramique se montre bien plus efficace : les grains se renouvellent rapidement et l'outil garde son mordant tout au long du travail. De même, on observe des qualités de matériaux différentes entre les outils d'aiguisage et les abrasifs proprement dits. Les outils d'aiguisage possèdent généralement un grain et un grade plus élevés. Il est ainsi possible d'utiliser ce critère pour distinguer des outils dont la morphologie proche rend parfois difficile l'identification fonctionnelle.

Car, plus que la morphologie, c'est avant tout le fonctionnement des outils qui diffère. Au sein de chaque catégorie fonctionnelle, on observe des modes de fonctionnement différents qui permettent de caractériser différents types d'outils. Au sein des abrasifs, on distingue trois types d'outils : les abraseurs, mobiles et actifs, les tablettes abrasives, mobiles et passives, et les tables abrasives, fixes et passives [ill. 3]. De même, les outils d'aiguisage se répartissent entre les aiguisoirs, mobiles et passifs sans zone de préhension, les affiloirs, mobiles et passifs avec zone de préhension à l'extrémité, les pierres à faux, mobiles et actives, et les meulets, fixes et passifs [ill. 4].

En contexte de travail des métaux, les outils d'aiguisage sont rares et se limitent à des formes de meulets de grande dimension, portant généralement de profondes traces d'utilisation. Ils sont utilisés pour l'émoulage. Au contraire, les abrasifs sont fréquents, pour ne pas dire systématiques. Une attention particulière doit être portée aux tables abrasives, en prélevant les sédiments autour de l'outil, qui peuvent contenir les restes du métal travaillé. Si ces outils forment la catégorie fonctionnelle la mieux documentée à ce jour, de nombreux points restent encore à éclaircir. Les abrasifs connus sont trop grossiers pour permettre le polissage. Cela implique nécessairement l'utilisation d'abrasifs libres, sous forme de poudre, dont nous ne connaissons pas encore la nature (cendres ou minéraux broyés).

\section{Les brunissoirs}

Les brunissoirs, comme les abrasifs, sont utilisés pour le surfaçage du métal [ill. 5]. Contrairement aux seconds, ils ne fonctionnent pas par enlèvement de matière mais par déformation plastique, en faisant subir au métal un frottement sous pression. Morphologiquement, ces outils se présentent sous forme de pierres de dimensions variables, mais offrant toujours des surfaces actives parfaitement lisses, afin d'éviter 


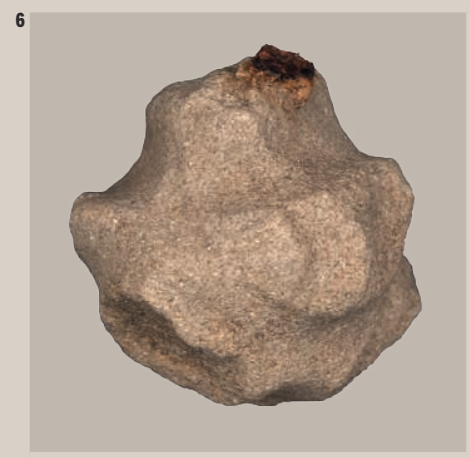

Demi-produit rond
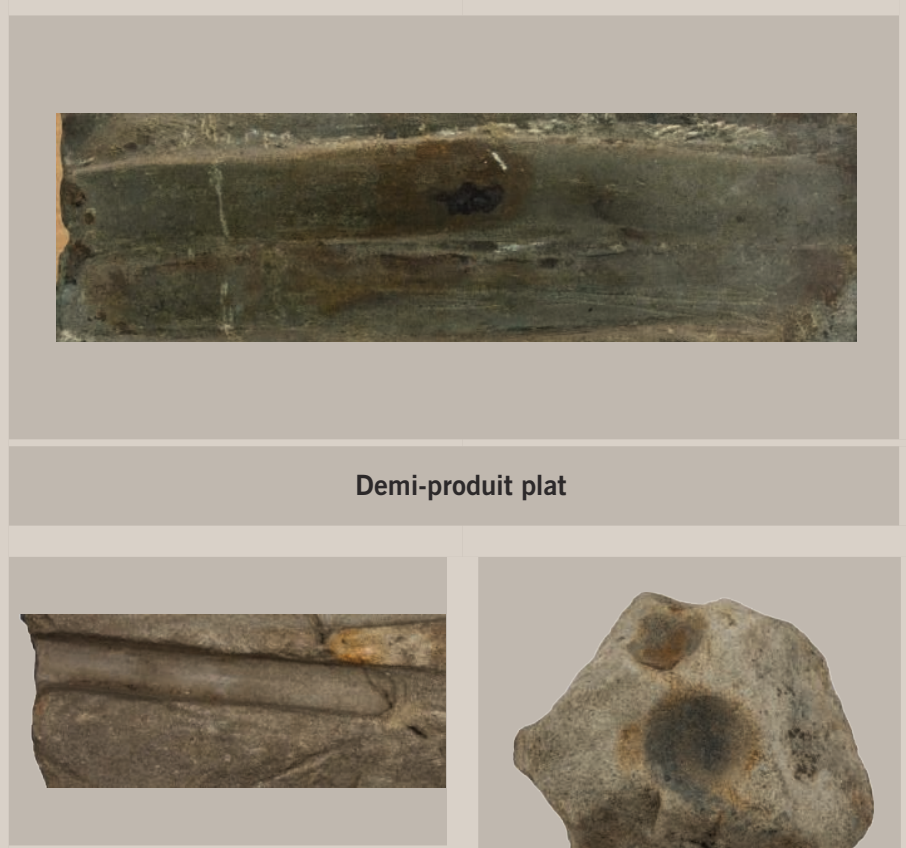

Demi-produit carré

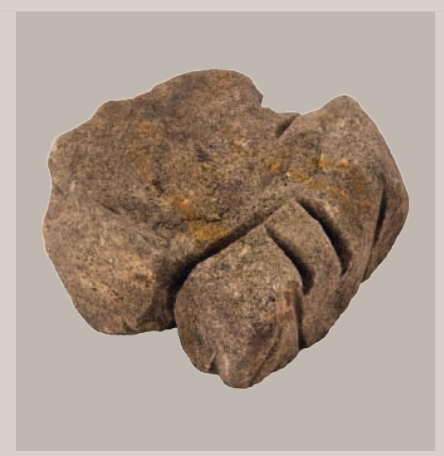

Ébarbage

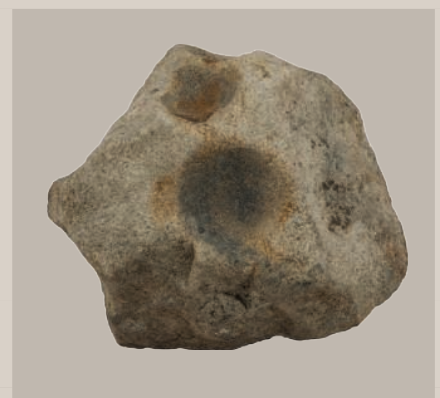

Objet hémisphérique

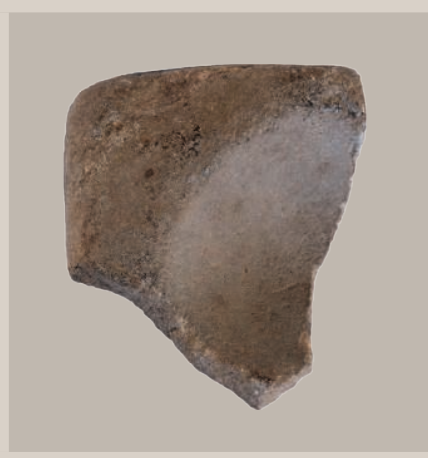

Objet hémisphérique

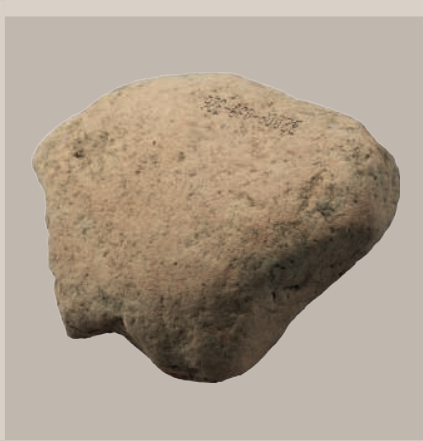

Anneau

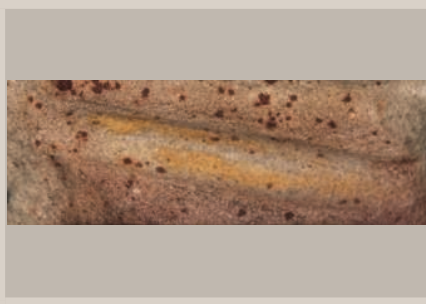

Résidus de fer

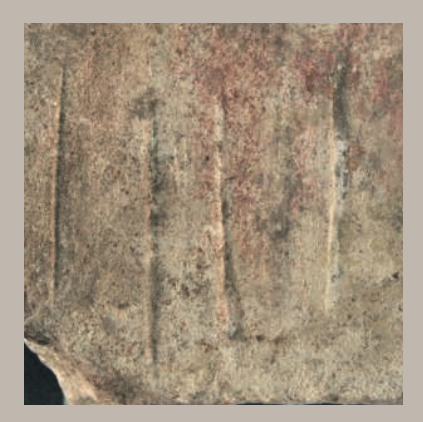

Aiguisage de pointe

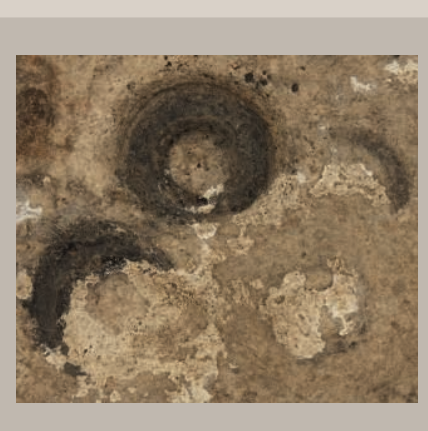

Objet concave

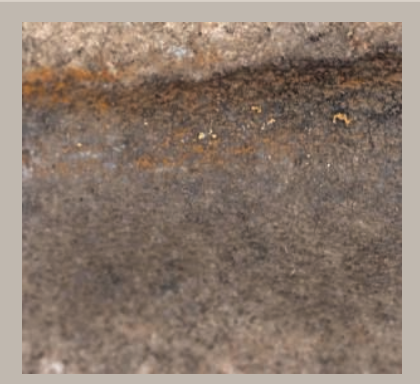

Résidus de fer et d'or 
la création de défauts sur le métal. Le matériau utilisé est toujours d'une dureté importante, à grains très fins, ne présentant pas d'abrasivité apparente. Les plus couramment utilisés sont le silex et la silexite, ainsi que la marcassite. La forme des surfaces actives est variable, généralement convexe, ce qui permet de limiter au maximum la surface de contact avec la matière, donc d'accentuer la pression.

\section{Les outils de broyage}

Les outils de broyage en contexte de transformation des métaux sont quasi exclusivement des broyons. La principale difficulté posée par ces outils est qu'ils sont également utilisés pour les activités culinaires. Toutefois, l'analyse de leur structure permet de distinguer aisément les deux types d'outils.

Aucune différence morphologique n'est perceptible entre les broyons techniques et culinaires. Seul le rayon de courbure des surfaces actives change et il est uniquement lié à celui du mortier avec lequel le broyon travaille. Aucune corrélation entre le domaine d'activité et le profil du broyon n'a pu être mise en évidence à ce jour. En revanche, on observe une différence significative dans la qualité de la surface active. Celle-ci est systématiquement lisse et unie pour les outils culinaires. Au contraire, les broyons techniques présentent généralement des surfaces rugueuses. Leur mode de fonctionnement diffère également. Les broyons culinaires sont utilisés uniquement en friction. Les broyons techniques sont utilisés à la fois en friction et en percussion, pour concasser le matériau avant de le broyer.

Techniquement, il semble que ces outils soient utilisés pour le broyage de matières minérales et végétales utilisées dans diverses opérations : dégraissants pour les tuyères et les creusets, fondants et mélanges de terres employés pour la trempe différentielle (la terre forme un isolant limitant l'échange thermique et les effets de la trempe sur une partie de la pièce produite). Une approche expérimentale devrait permettre de mieux comprendre cette fonction, avec un double obstacle. D'une part, il est encore difficile de réunir les couples broyons/mortier, généralement étudiés par des spécialistes différents, alors que l'on peut considérer qu'il s'agit d'un seul outil, comme la meta et le catillus d'une meule rotative. La seconde difficulté est que les matériaux utilisés pour fabriquer broyons et mortier varient, ce qui multiplie le nombre d'expérimentations à réaliser. Il s'agit d'un programme de recherche à part entière, encore inexploré.

\section{Tracéologie}

La restitution de la fonction d'un outil n'est qu'une première étape de son analyse. La suivante est d'en analyser l'usage. Outre l'analyse du contexte d'utilisation, qui varie en fonction de la structure de production et ne peut être abordé ici, l'approche tracéologique apporte un complément d'information important. Elle permet, grâce aux traces portées par un outil, d'en préciser l'usage. Cet aspect est secondaire pour des outils comme les supports de frappe, mis à part quelques cas anecdotiques. Il est en revanche particulièrement important pour les abrasifs et les outils d'aiguisage. Pour ces deux catégories fonctionnelles, il permet de restituer sinon les productions, du moins la forme des demi-produits employés lors des phases de production.

\section{Les abrasifs}

Le frottement d'un objet sur un abrasif, ou inversement d'un abrasif sur un objet, crée une empreinte dans l'abrasif [ill. 6]. La forme de cette empreinte renseigne sur celle de la pièce travaillée. Dans le meilleur des cas, la forme complète de l'objet peut être restituée, mais, généralement, seul le profil de l'objet peut l'être. Il correspond à la forme du demi-produit - forme intermédiaire entre la matière première (lopin, lingot) et l'objet fini - dont est issu l'objet. Cinq types de demiproduits peuvent être reconnus : plat, carré, rond, tôle et fil.

En fonction du profil longitudinal de la trace d'utilisation, il est possible de déterminer une longueur approximative de la pièce travaillée. Si la gorge est concave longitudinalement, cela désigne une pièce de faible longueur. La forme des extrémités de la gorge permet de mettre en évidence les pièces appointées.

Cette approche tracéologique est importante, même si elle ne permet que rarement de déterminer une production précise. Car même avec un niveau de détermination limité aux formes de demi-produits, elle peut mettre en évidence le travail de pièces pour lesquelles aucun indice métallique n'a pu être retrouvé. C'est le cas notamment des éléments de section importante, pour lesquels il est rare de retrouver des chutes, qui peuvent être réutilisées ou recyclées. Nous avons ainsi pu mettre en évidence une activité de forge lourde sur le site du Lycée militaire, qu'aucun outil ou fabricat métallique n'avait permis d'identifier.

\section{Les outils d'aiguisage}

Certains outils d'aiguisage sont conçus pour un seul type de tranchant. Les pierres à faux, comme l'indiquent leur nom, sont spécifiquement conçues pour l'aiguisage des faux (en réalité de tous les tranchants courbes). De même, aiguisoirs et affiloirs ne sont utilisés que pour l'aiguisage des lames courtes longitudinales (couteaux ou éventuellement poignards). Le cas du meulet est différent. Il peut être utilisé pour l'aiguisage de la plupart des tranchants et est indispensable pour les outils à tranchant transversal (ciseaux à bois par exemple). Les traces d'utilisation 
7. Traces d'aiguisage de lames

longues (a) et courtes (b)

sur des meulets provenant

des fouilles du 17, rue de

la Voie Romaine et du 14

route d'Étais à Entrains-

sur-Nohain (dir. Stépha

Venault, Inrap, étude

en cours).

$\Phi$
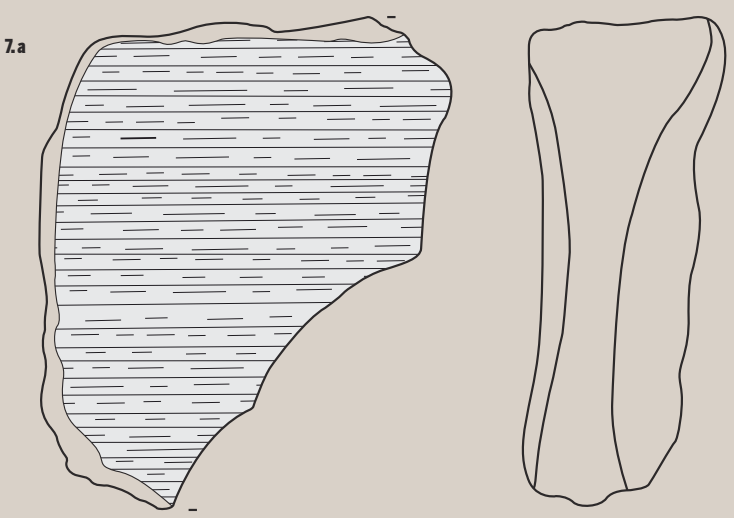

7.b
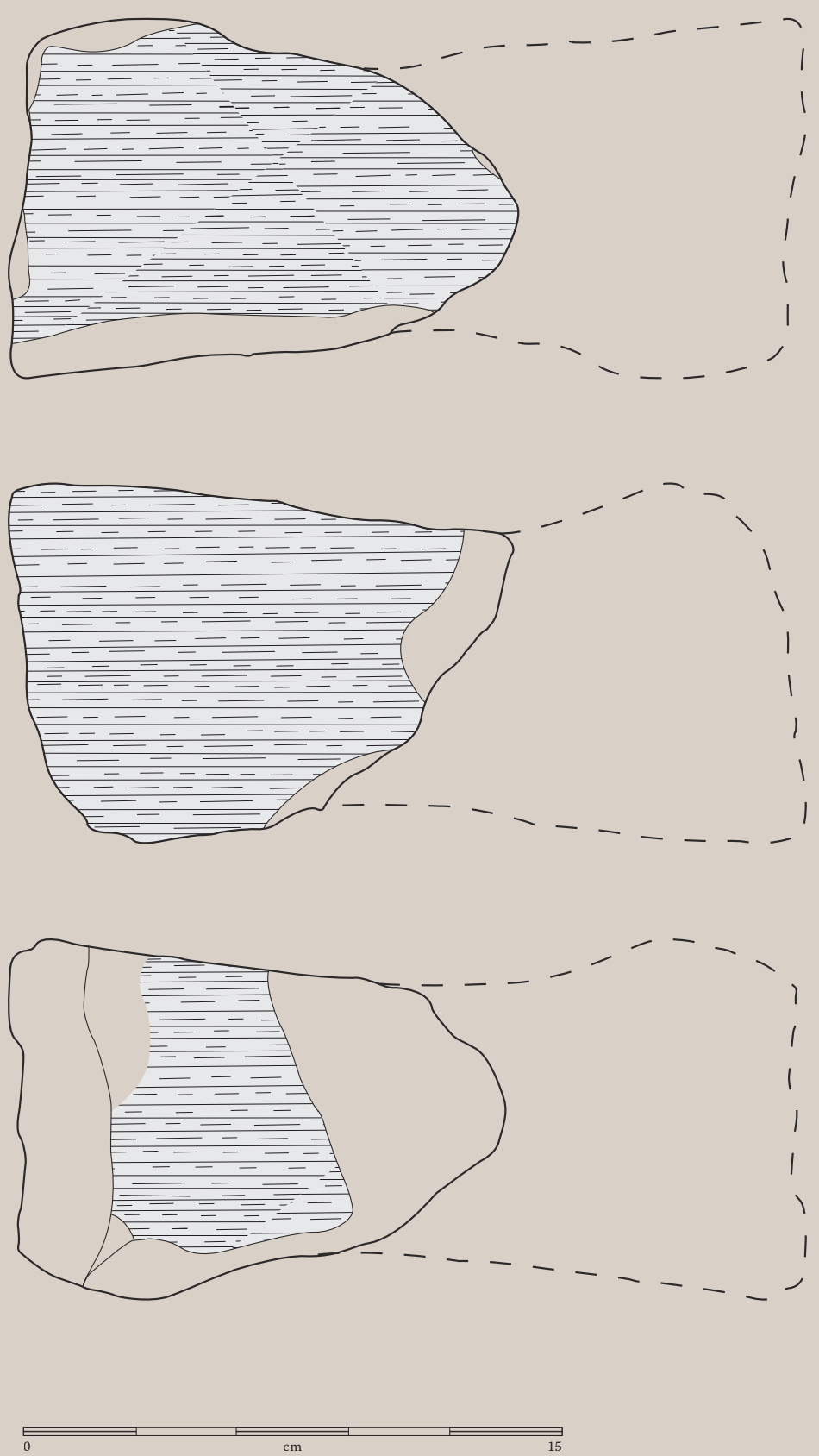
permettent néanmoins d'établir si l'outil aiguisé est à tranchant longitudinal ou transversal [ill. 7].

Dans le premier cas, toute la largeur de l'outil subit le frottement de la lame. La surface active se creuse uniquement dans le sens longitudinal et reste plane, ou peut devenir très légèrement convexe dans le sens transversal. On observe également dans certains cas une usure plus prononcée sur un côté, correspondant à la main directrice. Dans le cas de figure de l'aiguisage d'un outil à tranchant transversal, la zone la plus sollicitée par le travail est au centre de l'outil. La surface active se creuse ainsi progressivement d'une cuvette. Une courbure trop prononcée rend l'outil impropre à l'utilisation.

L'outillage lithique possède deux caractéristiques particulièrement intéressantes : ses qualités de conservation et l'absence de recyclage, qui en font une source de documentation de premier ordre, là où les outils et fabricats métalliques sont généralement absents ou corrodés. La méthodologie décrite ici permet de s'assurer d'un prélèvement optimum sur le terrain et d'extraire un maximum d'information des outils avérés. Néanmoins, en dehors de l'apport immédiat de ce mobilier pour la compréhension des techniques et la restitution des productions, d'autres perspectives se font jour. L'analyse des provenances des roches utilisées sur un site donné permet d'envisager de restituer la gestion de l'environnement d'un site (exploitation des ressources lithiques) et l'organisation des circuits d'échanges pour les pierres importées (notamment la pierre ponce). De même, l'analyse des propriétés mécaniques des roches devrait permettre à terme de mieux comprendre les choix opérés lors de la conception des outils et ainsi de mieux appréhender la connaissance que leurs concepteurs pouvaient avoir des matériaux. Car il est évident que l'utilisation de chaque pierre relève d'un choix réfléchi et non d'une sélection opportuniste. Les recherches sur l'outillage lithique n'en sont quà leur début et une prise en charge systématique de ce mobilier, jusqu’à présent négligé, ne peut qu'ouvrir des perspectives importantes dans le domaine de la métallurgie de transformation.
Références bibliographiques

BEAUNE S. A. de, 2008, L'homme et l'outil. L'invention technique durant la Préhistoire, Paris, CNRS éditions, 163 p.

Bluff L. A., Weir A. A. S., Rutz C., Wimpenny J. H., KACELNIK A., 2007, « Tool-related Cognition in New Caledonian Crows ", Comparative cognition E behavior reviews, vol. 2, p. 1-25.

Chardron-Picault P., Pernot M., 1999, Un quartier antique d'artisanat métallurgique à Autun (Saône-et-Loire). Le site du Lycée militaire, Paris, Maison des sciences de l'Homme, 316 p.

Guillaumet J.-P., 1996, L'artisanat chez les Gaulois, Paris, Errance, $127 \mathrm{p}$

JACOBI G., 1974, Werkzeug und Gerät aus dem Oppidum von Manching, Wiesbaden, Franz Steiner Verlag, $368 \mathrm{p}$.

LANDRIN H., 1835, Manuel du coutelier. Traité théorique et pratique de l'art de faire tous les ouvrages de coutellerie, Paris, Librairie encyclopédique Roret, $431 \mathrm{p}$

Mohen J.-P., Bailloud G., 1987, La vie quotidienne, Les fouilles du Fort-Harrouard, Paris, Picard (coll. L'âge du Bronze en France, 4), 241 p.

Pieters M., 2010, « Les supports de frappe lithiques pour le travail des métaux aux âges du Fer et à l'époque romaine », in Avec les archéologues Ardennais, Charleville-Mézières, Centre ardennais de recherche archéologique, bulletin $n^{\circ} 6$, p. 21-29.

Pieters M., 2013, Les outils comme traceurs des activités de transformation des métaux? Supports de frappe, abrasifs et brunissoirs, outils d'aiguisage et outils de broyage, Thèse de doctorat, Dijon, université de Bourgogne, $172 \mathrm{p}$. 